\title{
Children Conform to the Behavior of Peers; Other Great Apes Stick With What They Know
}

Psychological Science

2014, Vol. 25(12) 2160-2167

() The Author(s) 2014

Reprints and permissions:

sagepub.com/journalsPermissions.nav

DOI: $10.1177 / 0956797614553235$

pss.sagepub.com

\author{
Daniel B. M. Haun ${ }^{1,2,3}$, Yvonne Rekers ${ }^{1}$, and \\ Michael Tomasello ${ }^{1}$ \\ ${ }^{1}$ Max Planck Institute for Evolutionary Anthropology, Leipzig, Germany; ${ }^{2}$ Max Planck Institute for \\ Psycholinguistics, Nijmegen, The Netherlands; and ${ }^{3}$ Department of Developmental Psychology, \\ University of Jena
}

\begin{abstract}
All primates learn things from conspecifics socially, but it is not clear whether they conform to the behavior of these conspecifics-if conformity is defined as overriding individually acquired behavioral tendencies in order to copy peers' behavior. In the current study, chimpanzees, orangutans, and 2-year-old human children individually acquired a problem-solving strategy. They then watched several conspecific peers demonstrate an alternative strategy. The children switched to this new, socially demonstrated strategy in roughly half of all instances, whereas the other two great-ape species almost never adjusted their behavior to the majority's. In a follow-up study, children switched much more when the peer demonstrators were still present than when they were absent, which suggests that their conformity arose at least in part from social motivations. These results demonstrate an important difference between the social learning of humans and great apes, a difference that might help to account for differences in human and nonhuman cultures.
\end{abstract}

\section{Keywords}

culture, social learning, great apes, development

Received 6/5/13; Revision accepted 8/11/14

Human beings have created a variety of distinct cultural groups, each with its own ways of doing things. For a long time it was thought that no other animal species had anything resembling human culture. But detailed and long-term studies of various animal species in their natural habitats have established that they, too, form distinct social, or perhaps cultural, groups with multiple behavioral differences (Laland \& Galef, 2009). Arguably, some of the most detailed and convincing evidence for nonhuman culture-defined as socially acquired, populationspecific traits (Perry, 2006)—comes from the study of two of humans' closest phylogenetic relatives, chimpanzees (Luncz, Mundry, \& Boesch, 2012; van Leeuwen, Cronin, Haun, Mundry, \& Bodamer, 2012; Whiten et al., 1999) and orangutans (Krützen, Willems, \& van Schaik, 2011; van Schaik et al., 2003). But it is still unclear how similar the processes of culture and cultural transmission are in humans and these other great-ape species. Studies have shown, for example, that human children, chimpanzees, and orangutans can socially learn from conspecifics across transmission chains or via open diffusion in multiple steps, both of which are important components of cultural transmission (Dindo, Stoinski, \& Whiten, 2011; Flynn \& Whiten, 2008; Whiten, Horner, \& de Waal, 2005).

But humans do not just learn from other people, they adjust their responses to those of their peers (Asch, 1956; Bond \& Smith, 1996; Jenness, 1932), an effect dubbed conformity (Asch, 1956; Cialdini \& Goldstein, 2004; Haun, van Leeuwen, \& Edelson, 2013) or strong conformity (Haun \& Tomasello, 2011). Adjusting behavior to align with other people's, irrespective of the underlying

\footnotetext{
Corresponding Author:

Daniel B. M. Haun, Department of Developmental Psychology,

University of Jena, Am Steiger 3/1, D-07743 Jena, Germany

E-mail: daniel.haun@uni-jena.de
} 


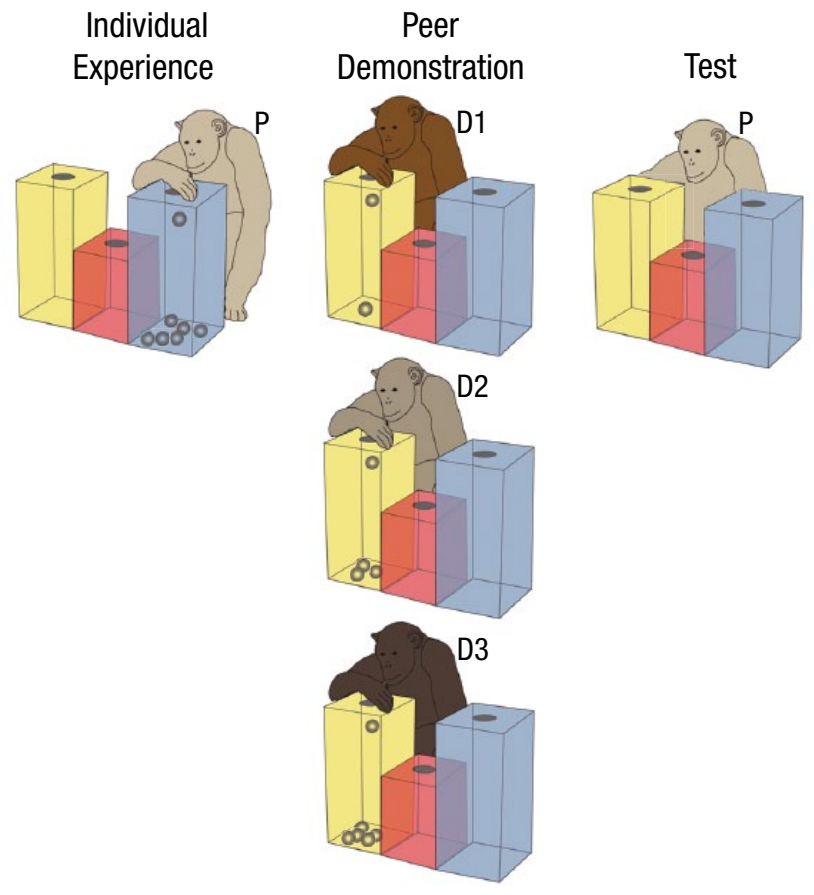

Fig. 1. Procedure in Study 1. Each participant (P) dropped balls into three holes in a puzzle box (left), one of which was associated with a reward. After the participant had demonstrated knowledge of which hole yielded rewards, a group of three peers (D1, D2, D3) demonstrated a successful alternative solution (middle). After the demonstration, the participant was given three balls, one at a time, and allowed to drop them into whichever holes he or she chose (right). The purpose of the test was to see whether participants would drop the ball in the same hole they had previously found to be associated with rewards or whether they would choose the hole the demonstrators had used.

motivation, is considered to be one of the driving forces behind cultural diversification (Boyd \& Richerson, 1985; Henrich \& Boyd, 1998). In cases in which peer influence leads individuals to abandon idiosyncrasies and to converge on the most prevalent conventions, groups move toward behavioral homogeneity while increasing betweengroups heterogeneity - the hallmark of culture (Richerson \& Boyd, 2004). This kind of social influence has been extensively studied in adults (Bond \& Smith, 1996), teenagers (Steinberg \& Monahan, 2007), and school-age children (Berenda, 1950). The available data on peer influence in children under the age of 10 remains comparatively sparse (Haun et al., 2013). To date, the youngest children shown to adjust their responses to a group of peers were 4 years of age (Haun \& Tomasello, 2011; Walker \& Andrade, 1996).

In recent years, in an attempt to reconstruct the evolutionary history of peer influence, several studies have investigated the structure of social learning in human's closest living relatives: the other great apes. Even though some studies have led authors to claim that nonhuman primates display humanlike conformity (Hopper, Schapiro,
Lambeth, \& Brosnan, 2011; Whiten et al., 2005), other researchers consider the case unsolved (Haun et al., 2013; van Leeuwen \& Haun, 2013). No previous study has directly compared humans' and nonhumans' tendencies to adjust their behavior to that of their peers in a single, comparable scenario.

\section{Study 1: Peer Influence Across Species}

In Study 1, we compared 2-year-old children's, chimpanzees', and orangutans' tendencies to abandon an individually acquired behavioral strategy after being exposed to a majority of peers demonstrating an equally effective alternative strategy.

\section{Metbod}

Participants. We analyzed a final sample of 18 children (Homo sapiens; 9 female, 9 male; mean age $=28$ months, $S D=3$, range $=24-33$ ), 12 chimpanzees (Pan troglodytes; 7 female, 5 male; mean age $=121$ months, $S D=54$, range $=72-252$ ), and 12 orangutans (Pongo pygmaeus; 6 female, 6 male; mean age $=102$ months, $S D=28$, range $=60-144)$. The planned goal of 18 participants per species was not met because of a lack of available animals (see the Supplemental Material available online for further participant details).

Procedure. The experimental setup consisted of a box with three sections, each of which was a different color. The sections were arranged horizontally; the two outer sections were the same height, whereas the middle section was shorter. In the top of each section was a hole (see Fig. 1). The box was attached to the outside of the steel mesh of an observation room in the case of the chimpanzees and orangutans, and it was placed on the ground for human children. When a ball was dropped in one of the holes, a reward was dispensed out of the bottom of that box. We controlled which holes had active triggers. Rewards were peanuts for chimpanzees and orangutans, and chocolate drops for human children. These foods were all highly desirable to participants, which minimized differences in food motivation across species.

During the initial phase, participants learned that all sections were open for ball insertions, but only one would yield rewards. They were allowed to drop balls in the sections until they used the assigned colored section in 8 out of 10 consecutive trials. Subsequently, participants were led to a location approximately $2 \mathrm{~m}$ from the box, where they watched while three familiar conspecific peers (demonstrators) interacted with the box one after the other. For chimpanzees and orangutans, participants and demonstrators were additionally separated by steel 


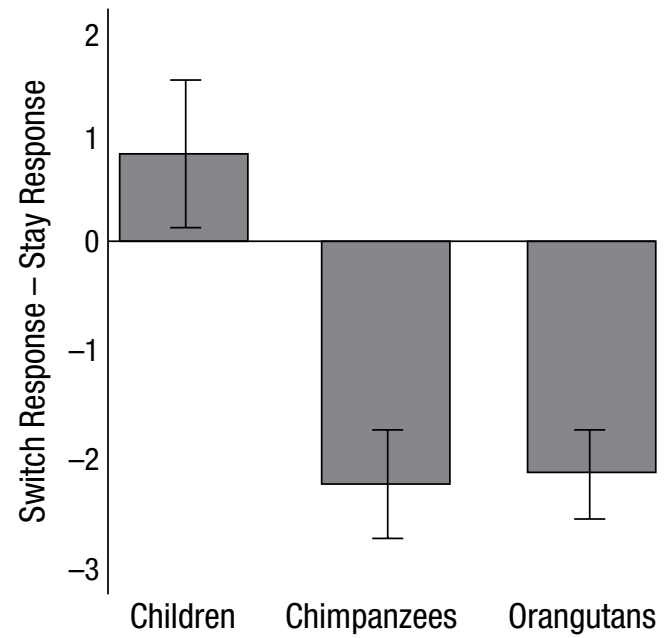

Fig. 2. Results from Study 1: average difference between switch and stay responses across groups. Error bars represent standard errors of the mean.

mesh. From their position, all participants had a clear line of sight to the box and could observe both the actions of the demonstrator and the dispensing of rewards.

The demonstrators had been previously trained to strongly prefer one of the box's three sections. The three demonstrators, one after the other, all used the same section (different from the participant's preference) twice each, receiving one reward in return for every ball (Fig. 1). We used three demonstrators because previous studies with human adults have shown that an effective majority needs to include at least three peers (Bond \& Smith, 1996). Further increases in majority size increase the number of conforming responses; however, the effect is not proportional to the increase in majority size, but diminishes with the increasing number of confederates (Latané \& Wolf, 1981; Tanford \& Penrod, 1984). The sections the participants and the demonstrators were trained on were fully counterbalanced across individuals. Balls were handed to the demonstrators only when the participant was facing their direction. If a participant was not looking while a demonstrator dropped the ball in the box, the trial was repeated.

After all six demonstrations, the demonstrators were led to a location where they could watch the participant but could not interfere. After a brief delay following the last demonstration $(\sim 30 \mathrm{~s})$, the testing period began. Participants were given three balls, one at a time, and were allowed to put each ball into whichever section they chose. All three of their choices were rewarded. Each response was coded from video as switching to match the majority response (switch), staying with the participant's own preference (stay), or choosing the third option that was neither the demonstrated nor the individually preferred one (other). Individuals' choices were easily identifiable via video. Nevertheless, all data were coded twice by two independent coders to minimize coding error.

\section{Results}

To calculate individuals' tendencies to switch strategy to match that of their peers, we subtracted the number of stay responses from the number of switch responses, which resulted in a difference score that varied between -3 and +3 , with negative scores indicating a preference to stay with one's individually acquired strategy and positive scores indicating a preference to switch to the peer group's strategy. The presence of a third response option for all participants ensured the independence of the two primary measures. We tested whether the three species varied in their relative tendency to switch to the demonstrated strategy with a between-subjects analysis of variance (ANOVA) with the factor species (human, chimpanzee, orangutan).

Results showed that species differed in their relative tendency to adjust their behavior to that of their peers (humans: $M=0.83, S E=0.68$; chimpanzees: $M=-2.25, S E=0.51$; orangutans: $M=-2.17, S E=0.41), F(2,39)=9.45, p<.001$, $\eta_{p}^{2}=.33$. We found that our data deviated from assumptions of normally distributed and homogeneous residuals by visually inspecting a quantile-quantile (Q-Q) plot and the residuals plotted against fitted values. To verify that the ANOVA reported valid results despite the deviation from assumptions, we ran a generalized linear mixed model (Baayen, 2008) and found highly similar results (see the Supplemental Material). Hence, we are confident that our ANOVA results were valid. Post hoc tests revealed that children's tendency to switch exceeded that of the other two ape species, whose tendencies did not differ-humans vs. chimpanzees: $t(27.9)=3.64, p=.001$ (Bonferroni-corrected $p=.006)$; humans vs. Orangutans: $t(26.2)=3.80, p=.001$ (Bonferroni-corrected $p=.005$ ); chimpanzees vs. orangutans: $t(20.9)=-0.13, p=.90$ (Fig. 2).

Finally, we tested whether individuals of any one species significantly adjusted their response to match that of their peers. Because we trained all participants to a criterion of selecting the same section of the box on 8 out of 10 consecutive trials, we expected individuals to continue to give 2.4 stay responses in the test on average $((3 / 10) \times 8)$ in case the peer demonstration had no effect. We further expected the remaining responses to be distributed equally across the remaining switch (0.3) and other (0.3) options. The resulting baseline (switch trials - stay trials) was -2.1 . Although human children gave more switch responses than expected, $t(17)=4.33$, $p<.001$ (Bonferroni-corrected $p=.003$ ), chimpanzees and orangutans did not adjust their responses after peer demonstration—chimpanzees: $t(11)=-0.29, p=.77$; 

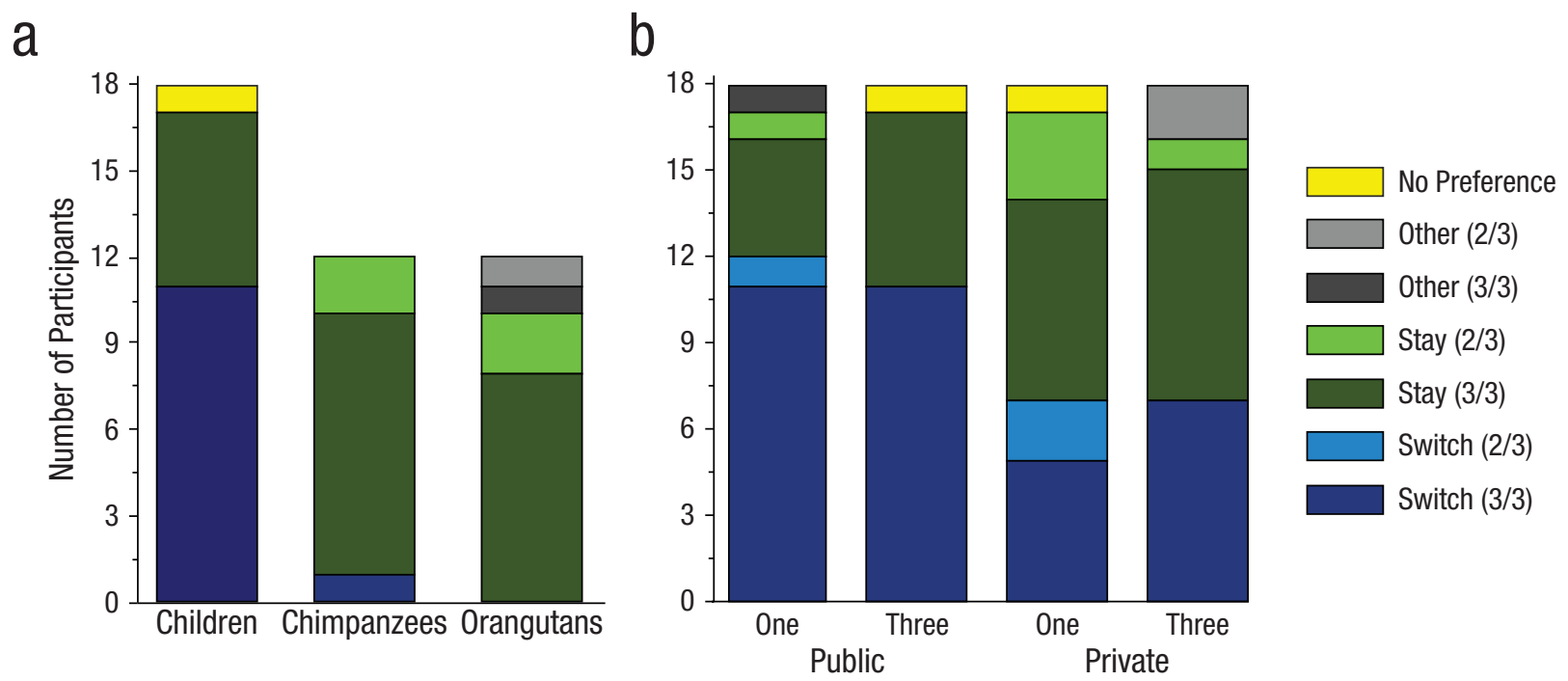

Fig. 3. Stacked bar graphs showing the distribution of participants according to their response pattern on test trials in (a) Study 1 and (b) Study 2. For Study 1, response patterns are shown for each group of participants. For Study 2, response patterns are shown across different levels of privacy (public response vs. private response) and numbers of demonstrators (one vs. three). Participants were categorized on the basis of whether they switched to a new section on two or three trials, stayed with the section they originally chose on two or three trials, or selected neither of these sections on two or three trials. In addition, a few participants showed no preference for any of the sections on the test trials.

orangutans: $t(11)=-0.16, p=.87$. The distribution of response patterns across all individuals in Study 1 can be seen in Figure 3a. On Trial 1, 12 of 18 children gave switch responses, 6 of 18 gave stay responses, and none gave other responses. Among chimpanzees, 2 of 12 gave switch responses, 10 of 12 gave stay responses, and none gave other responses on Trial 1. Among orangutans, 2 of 12 gave switch responses, 8 of 12 gave stay responses, and 2 of 12 gave other responses on Trial 1.

\section{Discussion}

Chimpanzees, orangutans, and 2-year-old human children observed conspecific peers displaying a behavioral strategy that was unlike their individually acquired strategy, though equal to it in effort and productivity. Children as young as 2 years of age were more likely to adjust their behavior to that of their peers than were either of the other two great-ape populations. When tested separately against expectation, only human children, but not the other two species, clearly adjusted their responses to match those of their peers. In fact, whereas human children conformed in over half of all instances, the two nonhuman great-ape populations almost exclusively stayed with their individually acquired strategies, ignoring the demonstrators, albeit both chimpanzees and children followed the majority when they acquired an entirely novel task and were therefore not required to adjust their behavioral preference (Haun, Rekers, \&
Tomasello, 2012). Although it is impossible to perfectly match experimental procedures across species, the present setup used peer demonstrators, identical apparatuses, and highly desirable food rewards across all species. In addition, only individuals demonstrating an understanding of the apparatus participated in the study.

Nevertheless, as always, alternative explanations are still possible. For example, greater general behavioral conservatism in the nonhuman great apes than in the human children (even in cases not involving social learning) might account for the differences (but see Lehner, Burkart, \& van Schaik, 2011; Van Leeuwen, Cronin, Schütte, Call, \& Haun, 2013; Manrique, Völter, \& Call, 2013 , for demonstrations of behavioral flexibility in great apes). Only future studies, accumulating results across various experimental designs and populations, and demonstrating greater conformity in human children than in nonhuman primates across a broader set of circumstances, can provide definitive evidence.

\section{Study 2: Dynamics of Peer Influence Among Children}

Social psychologists maintain a longstanding distinction between two interacting motivations for adjusting behavior to that of one's peer group in studies of conformity: behavioral optimization (informational conformity) and social strategizing (normative conformity; Deutsch \& Gerard, 1955). Informational conformists follow the majority position because it is a reliable source of information: 
Albeit not necessarily the best available option, the strategy used by most individuals is an aggregate of individual-learning outcomes and thus likely relatively safe, reliable, and productive. In support of this approach, mathematical models of evolutionary processes show that conforming to the majority-defined as the disproportionate likelihood of learners to adopt the behavior of the majority over alternatives - is adaptive, especially in variable environments with frequent migration between subpopulations, because it accelerates the acquisition of locally adaptive behaviors (Boyd \& Richerson, 1985; Henrich \& Boyd, 1998). In contrast, normative conformists follow the majority because of the social benefits of conforming relative to dissenting. Normative conformity is thus often driven by considerations of public appearance alone, whereas informational conformity often results in a genuine, socially mediated change in attitudes or even perception (Haun et al., 2013).

In Study 2, we tested for a similar distinction, assessing the relative contribution of normative and informational motivations for peer influence in 2 -year-old children. We assessed these two motivations by letting the children interact with the box either privately or publicly. Similar adjustment to peers in both the presence and absence of an audience suggests an informational influence, whereas stronger adjustment in the presence than in the absence of an audience suggests normative motivations. Furthermore, we were interested to see whether the number of peer demonstrators would affect children's tendency to adjust their behavior. Thus, in Study 2, we varied both the privacy of children's responses (public vs. private) and the number of demonstrators (one vs. three) to assess the composition of motivations underlying peer influence in 2-year-old children. Given prior studies reporting reduced levels of conformity in the absence of an audience in 4-year-old children (Haun \& Tomasello, 2011), we expected children to conform less when responding privately than when responding publicly. To our knowledge, no prior study on children's conformity manipulated the number of demonstrators, hence we did not have a priori expectations regarding the effect of the number of demonstrators.

\section{Metbod}

Participants. We analyzed a final sample of 72 children (36 female, 36 male; mean age $=28$ months, $S D=$ 2.2 , range $=24-33$; see the Supplemental Material for further participant details).

Procedure. We used the same general procedure and setup as in Study 1. Additionally, the procedure in Study 2 included four conditions in a $2 \times 2$ between-subjects design ( $n=18$ per condition), in which the size of the demonstrator group and the privacy of children's responses were varied. We varied the level of privacy by asking half of the children $(n=36)$ to respond in the presence of the demonstrators. For the other half ( $n=$ 36), the demonstrators left after the demonstration, leaving only the target child and the experimenter in the room. We varied the number of demonstrators such that half of each privacy group ( $n=18+18=36$ ) received demonstrations from a group of three peers, as in Study 1 , and the other half $(n=18+18=36)$ received a demonstration from one peer only.

\section{Results}

To calculate individuals' tendency to switch strategy to match their peers', we again subtracted the number of stay responses from the number of switch responses, which yielded a difference score that ranged between -3 and +3 , with negative scores indicating a preference to stay with one's individually acquired strategy and positive scores indicating a preference for an alternative strategy demonstrated by peers. We tested whether children's tendency to switch strategies varied using a $2 \times 2$ between-subjects ANOVA with the factors privacy (private vs. public) and number of demonstrators (1 vs. 3).

Children switched more when making their choices in the presence of the peer demonstrators $(M=1.0$, $S E=0.45)$ than in their absence $(M=-0.31, S E=0.44)$, $F(1,68)=4.17, p<.05, \eta_{p}{ }^{2}=.06$. Children switched equally often after a demonstration by three peers $(M=$ $0.31, S E=0.48)$ and by one peer only $(M=0.39, S E=$ $0.44), F(1,68)=0.02, p=.90$. We found no interaction between the two factors, $F(1,68$ ) $=0.15, p=.70$ (for descriptive statistics, see Fig. 4). We checked whether the assumptions of normally distributed and homogeneous residuals were fulfilled by visually inspecting a Q-Q plot and the residuals plotted against fitted values (both indicated no obvious deviations from these assumptions). We furthermore tested whether individuals in any one condition gave switch responses more than expected (baseline $=-2.1$ ). When responding publicly, children switched choices more than expectedone demonstrator: $t(17)=5.39, p<.0001$ (Bonferronicorrected $p=.0002)$; three demonstrators: $t(17)=4.33$, $p<.001$ (Bonferroni-corrected $p=.002$ ). When responding privately, children also switched choices more than expected-one demonstrator: $t(17)=2.85, p<.05$ (Bonferroni-corrected $p=.044)$; three demonstrators: $t(17)=2.81, p<.05$ (Bonferroni-corrected $p=.049)$. The distribution of responses across all individuals in Study 2 can be seen in Figure 3b. The distribution of responses in Trial 1 can be seen in Table 1 . 


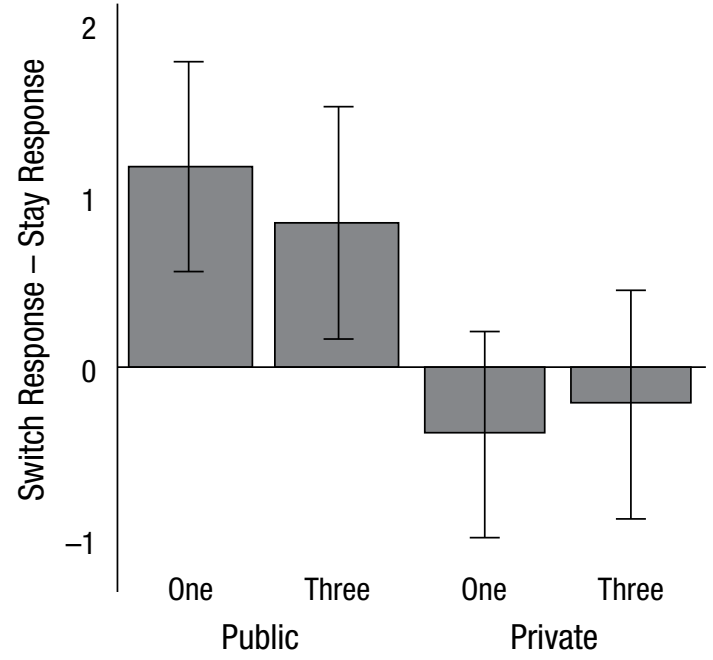

Fig. 4. Results from Study 2: average difference between switch and stay responses in 2-year-old human children across varying numbers of demonstrators (one vs. three) and levels of privacy (public response vs. private response). Error bars represent standard errors of the mean.

\section{Discussion}

The children in Study 2 appeared to adjust their behavior to that of their peers, in part out of social (normative) motivations: Children conformed more when tested in the presence of the demonstrators than in their absence. This replicates the results of a prior study in which the tendency of slightly older children (4-year-olds) to conform varied depending on whether they gave their response publicly or in private (Haun \& Tomasello, 2011); the similarity of these findings provides confidence in the present results, despite a rather small effect size. Whereas for adults, peer influence increases with a rising number of demonstrators (Latané \& Wolf, 1981; Tanford \& Penrod, 1984), 2-year-olds adjusted their behavior toward the demonstrated alternative just as readily when it was demonstrated by three other individuals than by one other individual, although children of the same age ( $M=2$ years, 3 months) have previously been shown to be sensitive to the difference in the number of demonstrators when they acquired an entirely novel task (Haun et al., 2012).
Many prior studies, both with children and with nonhuman animals, have suggested majority effects without experimentally demonstrating the specificity of those effects to situations involving a majority (van Leeuwen \& Haun, 2014). One important difference between the adult studies, which show an effect of the number of demonstrators on peer influence, and the present studies is that adults in most studies adjusted to their peers' preferences not only against their personal preference, as children in our studies did, but they also adopted a wrong opinion or a less-effective method under majority influence (Asch, 1956; Bond \& Smith, 1996; Cialdini \& Goldstein, 2004). Future studies should map out differences between those two scenarios.

\section{General Discussion}

We compared 2-year-old children's, chimpanzees', and orangutans' tendencies to abandon their behavioral preference in favor of matching the preference of a unanimous group of conspecific peers. We report three basic findings: (a) Children in our sample adjusted their behavior more readily than both chimpanzees and orangutans, who almost never adjusted their behavior to the majority's; (b) children adjusted their behavior at least in part for social motivations; and (c) children adjusted as much to just one peer as they did to a group of three.

Prior studies have allegedly shown peer conformity in chimpanzees (Hopper et al., 2011; Whiten et al., 2005). They have shown that chimpanzees revert back to the socially learned solution to open a puzzle box after discovering an equally effective alternative solution individually. Because the first, socially acquired, solution was also acquired by most group members, participants seemingly reverted back from a new innovation to the strategy that was demonstrated by most peers (Whiten et al., 2005). The interpretation of this reversion pattern, however, has since been criticized (Haun et al., 2013; van Leeuwen \& Haun, 2013). The most critical problem with the reversion design is that the socially learned strategy is always learned first, and the individual strategy is discovered only after the socially learned strategy has been adopted. It is thus impossible to identify a strategy of

Table 1. Results from Study 2: Distribution of Responses Across Varying Privacy Levels and Numbers of Demonstrators in Trial 1

\begin{tabular}{lccrrr}
\hline & \multicolumn{2}{c}{ Public response } & & \multicolumn{2}{c}{ Private response } \\
\cline { 2 - 3 } \cline { 5 - 6 } Response & One demonstrator & Three demonstrators & & One demonstrator & Three demonstrators \\
\hline Switch & 13 & 12 & & 13 & 9 \\
Stay & 4 & 0 & & 5 & 8 \\
Other & 1 & 0 & & 0 & 1 \\
\hline
\end{tabular}


reconvergence with the majority as either a case of peer influence or a primacy effect. Moreover, none of these prior studies required observers to change their individually acquired behavior to their peers' behavior. Rather, individuals were required to either stay with (Hopper et al., 2011) or revert back to (Whiten et al., 2005) the behavior they preferred before being exposed to the alternative.

We propose that the kind of conformity to peer influence documented in the present studies (the tendency to abandon a behavioral preference that was at least functionally equivalent in favor of matching the preference of peers) occurs in humans but not in chimpanzees or orangutans. We furthermore propose that this difference in susceptibility to peer influence between children and the other two great apes is driven by differences in social motivations: Children in our sample appeared to adjust their behavior, at least in part, because of the social consequences of seeming more or less similar to their peers. We propose that in the other two tested species, behavioral similarity across individuals does not in and of itself mediate social relationships to the same extent (Haun \& Over, 2013).

The possibility remains that chimpanzees and orangutans will conform to their peers' behavior under different circumstances. It has been reported, for example, that a chimpanzee female adjusted her behavior only several months after immigrating into a new group (Luncz \& Boesch, 2014). Because these scenarios are rare and difficult to test in a controlled setting, so far, only this single incidence can speak to this option. Finally, both sticklebacks and vervet monkeys have been shown to abandon their feeding preferences when exposed to a group of conspecifics with a different preference (Pike \& Laland, 2010; van de Waal, Borgeaud, \& Whiten, 2013). Although the motivations behind this behavior are unknown, the possibility remains that other animals adjust to peers in the same ways humans do.

Children's spontaneous proclivity to adjust their behavior to that of their peers provides them with a leg up for dealing with the many coordination problems inherent to human social life, such as norms and conventions (Lewis, 1969) and social interactions (Levinson, 2006). Furthermore, it promotes quick and stable in-group uniformity, stabilizing between-groups diversity (Boyd \& Richerson, 1985; Henrich \& Boyd, 1998). It has been argued that, in contrast to nonhumans, humans display a wider repertoire of behaviors that vary more distinctly across populations, of which a larger subsection is predominantly socially acquired (Pagel, 2012; Pagel \& Mace, 2004). Differences in peer influence across species, as documented in the present studies, might help explain these differences in the amount of cross-cultural variability between humans and other great-ape species.

\section{Author Contributions}

D. B. M. Haun, Y. Rekers, and M. Tomasello designed the study. D. B. M. Haun and Y. Rekers conducted the study. D. B. M. Haun analyzed the data. D. B. M. Haun and M. Tomasello wrote the manuscript.

\section{Acknowledgments}

We are grateful to L. Pharoah, R. Atencia, the Jane Goodall Institute, the Congolese Ministère de la Recherche Scientifique et de l'Innovation Technologique, and the staff of Tchimpounga Sanctuary, especially J. Maboto, B. Moumbaka, and L. Bibimbou. We also thank Biruté M. F. Galdikas, the Indonesian Ministry of Research and Technology, and the staff at Orangutan Foundation International's Orangutan Care Center and Quarantine, especially Pak Sehat, Ibu Waliyati, and Dr. Popo. We thank E. van Leeuwen and K. Liebal for comments and R. Mundry for statistical advice. Special thanks go to S. Schütte, S. Kennert, and N. Richter for their crucial help with organizing, collecting, and coding the data. In addition, we thank J. Dauksch, M. Missana, S. Taubert, N. Schumann, E. Kaminski, N. Biermann, A. Herbig, and $\mathrm{M}$. Weissbach for assistance with data collection.

\section{Declaration of Conflicting Interests}

The authors declared that they had no conflicts of interest with respect to their authorship or the publication of this article.

\section{Funding}

This research was funded by the Max Planck Society for the Advancement of Science.

\section{Supplemental Material}

Additional supporting information can be found at http://pss sagepub.com/content/by/supplemental-data

\section{References}

Asch, S. E. (1956). Studies of independence and conformity: I. A minority of one against a unanimous majority. Psychological Monographs, 70(9), 1-70.

Baayen, R. H. (2008). Analyzing linguistic data: A practical introduction to statistics using $R$. Cambridge, England: Cambridge University Press.

Berenda, R. W. (1950). The influence of the group on the judgments of children: An experimental investigation. New York, NY: King's Crown Press.

Bond, R., \& Smith, P. B. (1996). Culture and conformity: A metaanalysis of studies using Asch's (1952b, 1956) line judgment task. Psychological Bulletin, 119, 111-137.

Boyd, R., \& Richerson, P. J. (1985). Culture and the evolutionary process. Chicago, IL: University of Chicago Press.

Cialdini, R. B., \& Goldstein, N. J. (2004). Social influence: Compliance and conformity. Annual Review of Psychology, 55, 591-621.

Deutsch, M., \& Gerard, H. B. (1955). A study of normative and informational social influences upon individual judgment. The Journal of Abnormal and Social Psychology, 51, 629-636. 
Dindo, M., Stoinski, T., \& Whiten, A. (2011). Observational learning in orangutan cultural transmission chains. Biology Letters, 7, 181-183.

Flynn, E., \& Whiten, A. (2008). Cultural transmission of tool use in young children: A diffusion chain study. Social Development, 17, 699-718.

Haun, D. B. M., \& Over, H. (2013). Like me: A homophilybased account of human culture. In P. J. Richerson \& M. Christiansen (Eds.), Cultural evolution (pp. 75-85). Cambridge, MA: MIT Press.

Haun, D. B. M., Rekers, Y., \& Tomasello, M. (2012). Majoritybiased transmission in chimpanzees and human children, but not orangutans. Current Biology, 22, 727-731.

Haun, D. B. M., \& Tomasello, M. (2011). Conformity to peer pressure in preschool children. Child Development, 82, 1759-1767.

Haun, D. B. M., van Leeuwen, E. J. C., \& Edelson, M. G. (2013). Majority influence in children and other animals. Developmental Cognitive Neuroscience, 3, 61-71.

Henrich, J., \& Boyd, R. (1998). The evolution of conformist transmission and the emergence of between-group differences. Evolution \& Human Behavior, 19, 215-241.

Hopper, L. M., Schapiro, S. J., Lambeth, S. P., \& Brosnan, S. F. (2011). Chimpanzees' socially maintained food preferences indicate both conservatism and conformity. Animal Behaviour, 81, 1195-1202.

Jenness, A. (1932). Social influences in the change of opinion. The Journal of Abnormal and Social Psychology, 27, 29-34.

Krützen, M., Willems, E., \& van Schaik, C. P. (2011). Culture and geographic variation in orangutan behavior. Current Biology, 21, 1808-1812.

Laland, K. N., \& Galef, B. G. (2009). The question of animal culture. Cambridge, MA: Harvard University Press.

Latané, B., \& Wolf, S. (1981). The social impact of majorities and minorities. Psychological Review, 88, 438-453.

Lehner, S. R., Burkart, J. M., \& van Schaik, C. P. (2011). Can captive orangutans (Pongo pygmaeus abelii) be coaxed into cumulative build-up of techniques? Journal of Comparative Psychology, 125, 446-455.

Levinson, S. C. (2006). On the human "interaction engine." In N. J. Enfield \& S. C. Levinson (Eds.), Roots of human sociality: Culture, cognition and interaction (pp. 39-69). New York, NY: Berg.

Lewis, D. (1969). Convention: A philosophical study. Cambridge, MA: Harvard University Press.

Luncz, L. V., \& Boesch, C. (2014). Tradition over trend: Neighboring chimpanzee communities maintain differences in cultural behavior despite frequent immigration of adult females. American Journal of Primatology, 76, 649-657.

Luncz, L., Mundry, R., \& Boesch, C. (2012). Evidence for cultural differences between neighboring chimpanzee communities. Current Biology, 22, 922-926.
Manrique, H. M., Völter, C. J., \& Call, J. (2013). Repeated innovation in great apes. Animal Behaviour, 85, 195-202.

Pagel, M. (2012). Wired for culture: Origins of the human social mind. New York, NY: W. W. Norton.

Pagel, M., \& Mace, R. (2004). The cultural wealth of nations. Nature, 428, 275-278.

Perry, S. E. (2006). What cultural primatology can tell anthropologists about the evolution of culture. Annual Review in Anthropology, 35, 171-190.

Pike, T. W., \& Laland, K. N. (2010). Conformist learning in ninespined sticklebacks' foraging decisions. Biology Letters, 6, 466-468.

Richerson, P. J., \& Boyd, R. (2004). Not by genes alone: How culture transformed human evolution. Chicago, IL: University of Chicago Press.

Steinberg, L., \& Monahan, K. C. (2007). Age differences in resistance to peer influence. Developmental Psychology, 43, 1531-1543.

Tanford, S., \& Penrod, S. (1984). Social influence model: A formal integration of research on majority and minority influence processes. Psychological Bulletin, 95, 189-225.

van de Waal, E. V., Borgeaud, C., \& Whiten, A. (2013). Potent social learning and conformity shape a wild primate's foraging decisions. Science, 340, 483-485.

van Leeuwen, E. J. C., Cronin, K. A., Haun, D. B., Mundry, R., \& Bodamer, M. D. (2012). Neighbouring chimpanzee communities show different preferences in social grooming behaviour. Proceedings of the Royal Society B: Biological Sciences, 279, 4362-4367.

Van Leeuwen, E. J. C., Cronin, K. A., Schütte, S., Call, J., \& Haun, D. B. M. (2013). Chimpanzees (Pan troglodytes) flexibly adjust their behaviour in order to maximize payoffs, not to conform to majorities. PLOS ONE, 8(11), Article e80945. Retrieved from http://www.plosone.org/article/ info\%3Adoi\%2F10.1371\%2Fjournal.pone.0080945

van Leeuwen, E. J. C., \& Haun, D. B. M. (2013). Conformity in nonhuman primates: Fad or fact? Evolution \& Human Behaviour, 34, 1-7.

van Leeuwen, E. J. C., \& Haun, D. B. M. (2014). Conformity without majority? The case for demarcating social from majority influences. Animal Behaviour, 96, 187-194.

van Schaik, C. P., Ancrenaz, M., Borgen, G., Galdikas, B., Knott, C. D., Singleton, I., . . . Merrill, M. (2003). Orangutan cultures and the evolution of material culture. Science, 299, 102-105.

Walker, M. B., \& Andrade, M. G. (1996). Conformity in the Asch task as a function of age. The Journal of Social Psychology, 136, 367-372.

Whiten, A., Goodall, J., McGrew, W. C., Nishida, T., Reynolds, V., Sugiyama, Y., . . . Boesch, C. (1999). Cultures in chimpanzees. Nature, 399, 682-685.

Whiten, A., Horner, V., \& de Waal, F. B. M. (2005). Conformity to cultural norms of tool use in chimpanzees. Nature, 437 , 737-740. 